Conclusion We report the largest follow-up to-date of RTR from a single centre evaluating a novel, multicomponent transition programme. Our data indicates a longitudinal transition programme with a phased-handover via joint-clinics is positively associated with post-transfer renal allograft survival.

\section{G488(P) A NOVEL USE FOR ECULUZIMAB: SUCCESSFUL TREATMENT OF A CHILD WITH TRANSPLANT ASSOCIATED MICROANGIOPATHIC HAEMOLYTIC ANAEMIA}

C Kanaris, R West, R Wynn. Bone Marrow Transplant Unit, Royal Manchester Children's Hospital, Manchester, UK

10.1136/archdischild-2015-308599.441

Microangiopathic haemolytic anaemia (MAHA), as part of thrombotic microangiopathy (TMA) post bone marrow transplant, is well documented yet its management continues to challenge clinicians. Outside the transplant setting, classical thrombotic thrombocytopenia is often successfully treated with plasma exchange therapy, but there is evidence to suggest that is not beneficial for post-transplant patients particularly when acute graft-versus-host disease (GVHD) is present. Post-transplant TMA is characterised by MAHA, thrombocytopenia, elevated $\mathrm{LDH}$ and abnormal renal function. Fever and neurological impairment may also feature.

In children, management has mainly been supportive, with a focus on withdrawing risk factors. The aetiology is unconfirmed and is likely to be multifactorial. Infection, GVHD, irradiation and immunosuppressive drugs have been implicated as has the use of ATG in conditioning and CMV seronegativity. Outside of the transplant setting it is thought that inappropriate complement pathway is central to the development and treatment of TMAs and it has been hypothesised that complement dysregulation caused by ciclosporin may be responsible for some cases of post-transplant TMA.

Eculizumab is a monoclonal antibody that acts on the complement pathway by preventing the cleavage of C5 into C5a and $\mathrm{C} 5 \mathrm{~b}$, which forms part of a cytotoxic membrane attack complex. Eculizumab is licensed for use in adults and adolescents to treat atypical haemolytic uraemic syndrome and is the standard treatment for paroxysmal nocturnal haemoglobinuria in adults with emerging reports of its use to treat children with the same condition. There is also increasing evidence to support its use in children with atypical haemolytic uraemic syndromeand adult patients with TMA following renal transplant.
We present the case of an 18-month-old boy with Mucopolysaccharidosis Type 1 who following a bone marrow transplant, developed anaemia, thrombocytopenia, renal impairment with difficult to manage hypertension and raised LDH. A diagnosis of MAHA was reached and eculizumab was given after minimal improvement was seen after discontinuing ciclosporin. This resulted in an improvement in blood pressure and renal function, reduction in $\mathrm{LDH}$ and urine protein-creatinine ratio and reduced need for transfusion.

\section{G489(P) TREATMENT OF THE FIRST EPISODE OF CHILDHOOD IDIOPATHIC NEPHROTIC SYNDROME: A SYSTEMATIC REVIEW AND META-ANALYSIS}

${ }^{1}$ E Zolotas, ${ }^{2}$ Leontsinis. ${ }^{1}$ Paediatric Department, Abertawe Bro Morgannwg University Health Board, Swansea, UK; ${ }^{2}$ Renal Department, The Shrewsbury and Telford Hospital NHS Trust, Shrewsbury, UK

\subsection{6/archdischild-2015-308599.442}

Background and objective Corticosteroids induce remission in $80 \%$ of children with idiopathic nephrotic syndrome (INS). However $90 \%$ of steroid responders experience at least one relapse and $40 \%$ of them suffer from frequent relapses. The optimal treatment for the first episode of INS in terms of preventing subsequent relapses remains controversial.

Methods We conducted a systematic review and meta-analysis of randomised controlled trials (RCT). We searched MEDLINE, EMBASE and Cochrane Central Register of Controlled Trials without language restriction. We also searched proceedings from international conferences and we contacted investigators. We only included RCT which compared different regimens for the treatment of the first episode of INS in children.

Results 26 RCT were included. Nine studies compared the classic two-month steroid regimen with prolonged steroid courses ranging from three to seven months. Meta-analysis of those studies showed no difference in the number of children with frequent relapses [RR: 0.79 , 95\% CI $(0.57,1.08)$ ] (Figure 1) and number of relapses per patient [WMD: -0.37 , $95 \%$ CI $(-0.85,0.1)]$. Cumulative steroid dose was significantly higher with prolonged courses [SMD: 0.48 95\% CI $(0.16,0.81)]$ (Figure 2). Eight studies compared three months with six months of steroid treatment. Similarly there was no difference in frequent relapses [RR: 0.63 , 95\% CI $(0.36$, 1.10)] (Figure 3) and relapses per patient [WMD: $-0.32,95 \%$ CI $(-0.65,0.00)]$. Three RCT explored the potential benefit of adding cyclosporine, azithromycin or sarei-to, a Chinese herb,

\begin{tabular}{|l|lll}
\multicolumn{2}{c}{ Meta-analysis of studies comparing two-month steroid course with prolonged courses } \\
Outcome: Number of children with frequent relapses
\end{tabular}

Abstract G489(P) Figure 1 Meta-analysis of studies comparing two-month steroid course with prolonged courses 